\title{
A NOTE ON COMPACT SEMIDERIVATIONS
}

\author{
MATEJ BREŠAR \\ Department of Mathematics, University of Maribor \\ PEF, Koroška 160, 2000 Maribor, Slovenia \\ E-mail: bresar@uni-mb.si \\ YURI TUROVSKII
}

Institute of Mathematics and Mechanics of National Academy of Sciences of Azerbaijan F. Agaev St. 9, Baku AZ1141, Azerbaijan

E-mail: yuri@sinam.net

Dedicated to Professor Wiesław Żelazko on the occasion of his 70th birthday

\begin{abstract}
Let $\mathcal{A}$ be a Banach algebra without nonzero finite dimensional ideals. Then every compact semiderivation on $\mathcal{A}$ is a quasinilpotent operator mapping $\mathcal{A}$ into its radical.
\end{abstract}

1. Introduction. In [7] Ho proved that the existence of a compact derivation on $\mathcal{B}(H)$, the algebra of all bounded linear operators on a Hilbert space $H$, implies that $H$ is finite dimensional. This result has been extended to more general algebras of operators on Hilbert spaces in various ways $[1,8,9,11,15]$. In particular, compact derivations on von Neumann algebras, $C^{*}$-algebras, and nest algebras were described in [15], [1], and [11], respectively. On the other hand, Ho's result remains true in the algebra $\mathcal{B}(X)$ where $X$ is a Banach space. This is a simple consequence of the characterization of compact elementary operators on $\mathcal{B}(X)$ by Fong and Sourour [6] (cf. Example 1 in this paper) and the well-known fact that every derivation on $\mathcal{B}(X)$ is inner [4].

A natural question that now appears is what can we say about compact derivations in arbitrary Banach algebras. In fact, there are three related questions: what is the form and which are the properties of a compact derivation, what can be established about the space of all compact derivations, and what is the structure of a Banach algebra admitting nonzero compact derivations? We shall give some partial answers to these questions. Our main purpose, however, is to point out a method that is simple and elementary, but

2000 Mathematics Subject Classification: 47B47, 47B48, 47B07.

The first author is supported by MŠZŠ Grant P0-0501-0101.

The paper is in final form and no version of it will be published elsewhere. 
different from the methods used in the aforementioned papers. We intend to develop some ideas from this note in a subsequent, longer and more technical paper.

Our note also follows the line of investigation concerning the question when a derivation of a Banach algebra $\mathcal{A}$ has its range in the (Jacobson) $\operatorname{radical} \operatorname{rad}(\mathcal{A})$ of $\mathcal{A}$. It is well-known that in the case when $\mathcal{A}$ is commutative this holds true for every derivation [14], and there are various noncommutative extensions of this famous result (see for example Mathieu's survey [10] on this topic).

Yet another motivation for us are results on algebras having nonzero derivations of finite rank. For example, [2, Corollary 2] tells us that if such an algebra is prime then it must be finite dimensional, and [3, Corollary 5.5] gives an appropriate generalization of this assertion to semiprime algebras. Our results can be in particular viewed as analytic extensions of these purely algebraic results.

Let us point out some immediate observations concerning compact derivations, and thereby indicate what answers one can expect concerning the three questions posed above. First, if a Banach algebra $\mathcal{A}$ contains a finite dimensional ideal $\mathcal{I}$ and $D$ is a bounded derivation on $\mathcal{A}$ having its range in $\mathcal{I}$ (say, an inner derivation $x \mapsto a x-x a$ where $a \in \mathcal{I}$ ), then $D$ has finite rank and so in particular it is compact. In our main result we shall therefore confine ourselves to algebras without nonzero finite dimensional ideals. Next, if a bounded derivation $D$ has a nonzero eigenvalue $\lambda$, then every corresponding eigenvector $a$ is nilpotent. This is well-known and easy to see (just note that $n|\lambda| \cdot\left\|a^{n}\right\|=$ $\left\|D\left(a^{n}\right)\right\| \leq\|D\| \cdot\left\|a^{n}\right\|$ for every $\left.n \geq 1\right)$. Therefore, a compact derivation on a Banach algebra without nonzero nilpotent elements is necessarily a quasinilpotent operator. Now one might wonder whether the same is true under some milder assumptions than the non-existence of proper nilpotents. Finally we remark that compact derivations on $\mathcal{A}$ form a closed ideal of the Lie algebra of all bounded derivations on $\mathcal{A}$.

Our method works for maps more general than derivations. A linear map $D: \mathcal{A} \rightarrow \mathcal{A}$ is called a semiderivation [2] if there exists an algebra endomorphism $T$ on $\mathcal{A}$ such that $T D=D T$ and

$$
D(x y)=D(x) T(y)+x D(y)=D(x) y+T(x) D(y)
$$

for all $x, y \in \mathcal{A}$. Basic examples of semiderivations are derivations (for $T=1$ ) and maps of the form $D=1-T$ where $T$ is any algebra endomorphism. Note that a semiderivation $D$ satisfies

$$
L_{D(a)}=D L_{a}-L_{T(a)} D \quad \text { and } \quad R_{D(a)}=D R_{a}-R_{T(a)} D
$$

for every $a \in \mathcal{A}$; here, $L_{x}$ and $R_{x}$ denote the left and the right multiplications by $x$, i.e., $L_{x}: y \mapsto x y$ and $R_{x}: y \mapsto y x$.

\section{The results}

TheOREM 2.1. Let $\mathcal{A}$ be a Banach algebra without nonzero finite dimensional ideals. If $D$ is a compact semiderivation on $\mathcal{A}$, then $D(\mathcal{A}) \subseteq \operatorname{rad}(\mathcal{A})$ and $D$ is a quasinilpotent operator.

Proof. Suppose that $D(a) \notin \operatorname{rad}(\mathcal{A})$ for some $a \in \mathcal{A}$. Then there is $b \in \mathcal{A}$ such that the element $c=D(a) b$ is not quasinilpotent. Accordingly, the operators $L_{c}$ and $R_{c}$ are 
not quasinilpotent. Since $L_{c}=D L_{a b}-L_{T(a)} D L_{b}$ and $R_{c}=R_{b} D R_{a}-R_{T(a) b} D$, these two operators are compact. Therefore, each of them has a nonzero eigenvalue whose corresponding eigenspace is finite dimensional. Let $\lambda$ be a nonzero eigenvalue of $L_{c}$ and let $u \in \mathcal{A}$ be its eigenvector. Thus, $u \neq 0$ and $\left(L_{c}-\lambda\right) L_{u}=0$. This means that the range of $L_{u}$ is contained in the eigenspace and so $L_{u}$ has finite rank, i.e. the right ideal $u \mathcal{A}$ is finite dimensional. In order to show that $R_{u}$ has also finite rank we use dual operators. Note that $\left(R_{c}^{*}-\lambda\right) R_{u}^{*}=0$ with $R_{c}^{*}$ compact, whence $R_{u}^{*}$ is of finite rank by the same argument and so is $R_{u}$. That is, the left ideal $\mathcal{A} u$ is finite dimensional. But then the ideal $\mathcal{A} u \mathcal{A}$ is finite dimensional. Indeed, just note that if the sets $\left\{a_{1} u, \ldots, a_{n} u\right\}$ and $\left\{u b_{1}, \ldots, u b_{m}\right\}$ generate the vector spaces $\mathcal{A} u$ and $u \mathcal{A}$, respectively, then the set $\left\{a_{i} u b_{j} \mid i=1, \ldots, n, j=1, \ldots, m\right\}$ generates the space $\mathcal{A} u \mathcal{A}$. Thus, the ideal generated by $u, \mathbb{C} u+\mathcal{A} u+u \mathcal{A}+\mathcal{A} u \mathcal{A}$, is a nonzero finite dimensional ideal. This of course contradicts our initial assumption. Therefore, $D(\mathcal{A}) \subseteq \operatorname{rad}(\mathcal{A})$.

Now suppose that $D$ is not quasinilpotent. Without loss of generality we may then assume that 1 is its eigenvalue. Its corresponding eigenspace $\mathcal{V}$ is finite dimensional and, since $D$ and $T$ commute, it is invariant under $T$. So one can find a nonzero $v \in \mathcal{V}$ and $\mu \in \mathbb{C}$ such that $T(v)=\mu v$. Pick a spectral idempotent $P$ of $D$ such that $1-P$ is of finite rank and $r(D P)<\frac{1}{1+|\mu|}$ (here, $r($.$) denotes the spectral radius). Note that$ $D L_{v}-\mu L_{v} D=L_{v}$, and hence

$$
D P\left(P L_{v} P\right)-\mu\left(P L_{v} P\right) D P=P L_{v} P .
$$

That is, $\left(L_{D P}-\mu R_{D P}\right)\left(P L_{v} P\right)=P L_{v} P$. However, since

$$
r\left(L_{D P}-\mu R_{D P}\right) \leq r\left(L_{D P}\right)+|\mu| r\left(R_{D P}\right)=(1+|\mu|) r(D P)<1
$$

it follows that $P L_{v} P=0$. But then $L_{v}=(1-P) L_{v}+P L_{v}(1-P)$ has finite rank. Similarly we see that $R_{v}$ has finite rank. As shown above, this is impossible since it implies that the ideal generated by $v$ is finite dimensional.

Corollary 2.2. Let $\mathcal{A}$ be a Banach algebra without nonzero finite dimensional ideals. Then the closed associative algebra generated by all compact derivations on $\mathcal{A}$ consists of quasinilpotent operators.

Proof. Theorem 2.1 tells us that the Lie algebra of all compact derivations on $\mathcal{A}$ consists of quasinilpotent operators. Therefore our conclusion follows from [12, Corollary 11.6].

Suppose that $\mathcal{A}$ is a semiprime Banach algebra. If $\mathcal{I}$ is a nonzero finite dimensional ideal of $\mathcal{A}$, then $\mathcal{I}$ itself is a finite dimensional complex semiprime algebra, and as such, by Wedderburn theorem, it is isomorphic to $M_{n_{1}} \times \ldots \times M_{n_{s}}$ for some positive integers $n_{1}, \ldots, n_{s}$ (here, $M_{n}$ denotes the algebra of all $n \times n$ complex matrices). In particular, $\mathcal{I}$ has an identity element from which one easily infers that there is a central idempotent $e$ in $\mathcal{A}$ such that $\mathcal{I}=e \mathcal{A}$. The condition required in Theorem 2.1 can be therefore stated in a nicer form. Moreover, since prime algebras cannot contain central idempotents different from 0 and 1 , Theorem 2.1 yields the following result.

Corollary 2.3. Let $\mathcal{A}$ be an infinite dimensional prime Banach algebra. If $D$ is a compact semiderivation on $\mathcal{A}$, then $D(\mathcal{A}) \subseteq \operatorname{rad}(\mathcal{A})$ and $D$ is a quasinilpotent operator. 
We remark that there really exist nonzero compact derivations on infinite dimensional prime Banach algebras. For example, take a commutative prime Banach algebra $\mathcal{B}$ with compact regular representation on itself [5] (i.e., every $L_{b}, b \in \mathcal{B}$, is compact). Then $\mathcal{A}=M_{2}(\mathcal{B})$ is prime and inner derivations on $\mathcal{A}$ are compact.

Primitive algebras are both prime and semisimple. For these algebras Corollary 2.3 therefore gets a particularly simple form.

COROllary 2.4. Let $\mathcal{A}$ be a primitive Banach algebra. If there exists a nonzero compact semiderivation on $\mathcal{A}$, then $\mathcal{A}$ is finite dimensional.

Note that Corollary 2.4 in particular generalizes the result of Ho mentioned at the beginning. If we consider only derivations, then this corollary can be extended as follows.

Corollary 2.5. Let $D$ be a compact derivation on a Banach algebra $\mathcal{A}$. If $\mathcal{P}$ is a primitive ideal in $\mathcal{A}$ of infinite codimension, then $D(\mathcal{A}) \subseteq \mathcal{P}$. Accordingly, $D(\mathcal{A}) \subseteq \operatorname{rad}(\mathcal{A})$ in the case when $\mathcal{A}$ has no primitive ideals of finite codimension.

Proof. Since $\mathcal{P}$ is invariant under $D$ [13, Theorem 2.2], $D$ induces a derivation $D_{\mathcal{P}}$ on $\mathcal{A} / \mathcal{P}$ defined by $D_{\mathcal{P}}(x+\mathcal{P})=D(x)+\mathcal{P}$. Note that $D_{\mathcal{P}}$ is compact and so Corollary 2.4 implies that $D_{\mathcal{P}}=0$; that is, $D(\mathcal{A}) \subseteq \mathcal{P}$.

$C^{*}$-algebras admitting nonzero compact derivations were characterized in $[1$, Corollary 2.4]. We are now in a position to generalize this result to semisimple Banach algebras.

Corollary 2.6. Let $\mathcal{A}$ be a semisimple Banach algebra. Then there exists a nonzero compact derivation on $\mathcal{A}$ if and only if there exists a central idempotent $e$ in $\mathcal{A}$ such that the ideal $e \mathcal{A}$ is finite dimensional and noncommutative.

Proof. Suppose there exists a nonzero compact derivation on $\mathcal{A}$. As pointed out above, Theorem 2.1 implies that there is a nonzero central idempotent $e$ such that $e \mathcal{A} \cong M_{n_{1}} \times$ $\ldots \times M_{n_{s}}$. Moreover, from the proof of Theorem 2.1 we see that $e$ can be chosen so that the ideal $e \mathcal{A}$ is generated by an element $u$ in $D(\mathcal{A}) \mathcal{A}$ (namely, $u=\lambda^{-1} d(a) b u$ ). If every $n_{i}$ was 1 then $D$ would clearly vanish on $e \mathcal{A}$, and so $u=e u \in e D(\mathcal{A}) \mathcal{A}=D(e \mathcal{A}) \mathcal{A}=\{0\}$, a contradiction. Therefore $n_{i} \geq 2$ for some $n_{i}$, meaning that $e \mathcal{A}$ is noncommutative. The converse is trivial - just take an inner derivation implemented by a non-central element in $e \mathcal{A}$.

Of course one can choose $e$ in Corollary 2.6 so that $e \mathcal{A} \cong M_{n}$ for some $n \geq 2$. Let us also mention that Corollary 2.6 does not hold (at least not in the same form) for semiderivations. Namely, the algebra $\mathbb{C} \times \mathbb{C}$ has a nontrivial automorphism, and therefore there are nonzero semiderivations on this (commutative) algebra.

\section{References}

[1] C. A. Akemann and S. Wright, Compact and weakly compact derivations of $C^{*}$-algebras, Pacific J. Math. 85 (1979), 253-259.

[2] J. Bergen, Derivations in prime rings, Canad. Math. Bull. 26 (1983), 267-270.

[3] M. Brešar and D. Eremita, The lower socle and finite rank elementary operators, Comm. Algebra 31 (2003), 1485-1497. 
[4] P. R. Chernoff, Representations, automorphisms, and derivations on some operator algebras, J. Funct. Anal. 12 (1973), 275-289.

[5] H. V. Dedania, Compact elements in weighted discrete semigroup algebras, in: Banach Algebras 97, W. de Gruyter, Berlin, 1998, 119-134.

[6] C. K. Fong and A. Sourour, On the operator identity $\sum A_{k} X B_{k} \equiv 0$, Canad. J. Math. 31 (1979), 845-857.

[7] Y. Ho, A note on derivations, Bull. Inst. Math. Acad. Sinica 5 (1977), 1-5.

[8] J. Li, Compact and weakly compact derivations of certain CSL algebras, Taiwanese J. Math. 3 (1999), 243-249.

[9] M. Mathieu, Properties of the product of two derivations of a $C^{*}$-algebra, Canad. Math. Bull. 32 (1989), 490-497.

[10] M. Mathieu, Where to find the image of a derivation, Banach Center Publ. 30 (1994), 237-249.

[11] C. Peligrad, Compact derivations of nest algebras, Proc. Amer. Math. Soc. 97 (1986), 668-672.

[12] V. S. Shulman and Yu. V. Turovskii, Joint spectral radius, operator semigroups, and a problem of W. Wojtyński, J. Funct. Anal. 177 (2000), 383-441.

[13] A. M. Sinclair, Continuous derivations on Banach algebras, Proc. Amer. Math. Soc. 20 (1969), 166-170.

[14] M. P. Thomas, The image of a derivation is contained in the radical, Ann. Math. 128 (1988), 435-460.

[15] S.-K. Tsui, Compact derivations on von Neumann algebras, Canad. Math. Bull. 24 (1981), $87-90$. 